\title{
Color and spectral analysis of daylight in southern Europe
}

\author{
Javier Hernández-Andrés, Javier Romero, and Juan L. Nieves \\ Departamento de Óptica, Facultad de Ciencias, Universidad de Granada, Granada 18071, Spain \\ Raymond L. Lee, Jr. \\ Mathematics and Science Division, U.S. Naval Academy, Annapolis, Maryland 21402
}

Received August 4, 2000; revised manuscript received November 30, 2000; accepted December 18, 2000

\begin{abstract}
We have analyzed the colorimetric and spectral characteristics of 2600 daylight spectra (global spectral irradiances on a horizontal surface) measured for all sky states during a 2-year period at Granada, Spain. We describe in detail the chromaticity coordinates, correlated color temperatures (CCT), luminous efficacies, and relative UV and IR contents of Granada daylight. The chromaticity coordinates of Granada daylight lie far above the CIE locus at high CCTs ( $>9000 \mathrm{~K}$ ), and a CCT of $5700 \mathrm{~K}$ best typifies this daylight. Our principalcomponents analysis shows that Granada daylight spectra can be adequately represented by using sixdimensional linear models in the visible, whereas seven-dimensional models are required if we include the UV or near-IR. Yet on average only three-dimensional models are needed to reconstruct spectra that are colorimetrically indistinguishable from the original spectra. (C) 2001 Optical Society of America
\end{abstract}

OCIS codes: $010.1290,330.1710,330.1730$.

\section{INTRODUCTION}

Detailed knowledge of the spectral power distribution (SPD) of daylight in the visible, ultraviolet, and nearinfrared parts of the spectrum at different sites and for varying atmospheric conditions has many applications. Such knowledge is important in scientific disciplines (e.g., color vision, atmospheric optics, meteorology, medicine, biology, and materials research), technical areas (e.g., photovoltaic applications, biomass production, color rendering and metamerism, agriculture, and architecture), and in industry (e.g., photography, dyes, paints, and textiles).

During the 1960's and 1970's, measurement campaigns were conducted in several countries (United States, England, Japan, India, South Africa, Australia) in efforts to determine representative daylight spectra $E(\lambda) .^{1-14} \mathrm{Re}-$ searchers variously made these measurements using spectroradiometers, ${ }^{1-2,4-5,7-14}$ colorimeters, ${ }^{3}$ or visual color-temperature meters. ${ }^{6}$ Furthermore, their measurements involved different detector orientations, fields of view, and sky states, and they sometimes analyzed daylight and skylight data simultaneously. Despite this variety of techniques and equipment, these studies consistently reached two basic conclusions. First, daylight's different phases have chromaticities that lie near the Planckian locus of the 1931 chromaticity diagram adopted by the Commission Internationale de l'Eclairage (CIE). Second, these different daylight power spectra are highly correlated with one another, and this underlying similarity has many practical uses. However, none of these older studies include daylight spectra measured over both a wide range of atmospheric conditions and a long time period. For example, Condit and Grum mea- sured 251 spectra in just four weeks, ${ }^{4}$ and Tarrant measured only 391 spectra over four years. ${ }^{11}$

The paper by Judd et al., ${ }^{2}$ which analyzes three sets of daylight spectra, is the one most often cited in the literature and is the basis for the CIE's daylight recommendations. Notable among the results of Judd et al. is that most daylight SPDs can be accurately estimated by linearly combining three fixed basis functions. Although Judd et al. remains perhaps the best experimental analysis of daylight to date, its results depend heavily on the particular conditions under which its spectra were acquired, such as sky state, field of view, and detector orientation.

Other papers on daylight and skylight spectra have repeatedly shown, ${ }^{1-14}$ in agreement with Judd et al., that chromaticity provides good estimates not only of daylight's visible spectra but also of its correlated color temperature $(\mathrm{CCT})$. Such findings prompted the CIE to propose a method for calculating the relative SPD of typical daylight phases that uses only CCT values. ${ }^{15}$ When the spectral region is broad (including the near-IR, where there is lower spectrum-to-spectrum correlation), Slater and Healey show that highly accurate daylight spectra require linear models of seven dimensions rather than the CIE's three. ${ }^{16}$ In addition, Romero et al. use sevendimensional models to accurately reconstruct SPDs of both daylight and artificial illuminants. ${ }^{17}$

We have earlier noted the potentially confusing nature of daylight terminology, ${ }^{18}$ and others have attempted to eliminate this confusion. ${ }^{19}$ To avoid ambiguity, one must specify what is being measured: the natural-light component (skylight, direct sunlight, or their combination), radiometric variable (radiance or irradiance), and any 
other parameters which affect SPDs (e.g., weather, detector orientation, field of view, sampling interval). In addition, we follow Henderson's advice that it is better to analyze "homogeneous sets of observations for single locations than to lose the small differences by combining a large, heterogeneous collection of curves" (Ref. 20, p. 184).

Although daylight is essentially achromatic, many factors (e.g., solar elevation, site altitude, atmospheric conditions, pollution, detector orientation) can affect its spectral composition and thus its color. For our purposes, analyzing only the daylight spectra themselves runs the risk of hiding perceptually interesting facts, and this leads us to colorimetric analysis of our spectra. As Henderson recommends, we acquired daylight spectra over a long time period at one site, and this makes our colorimetry both novel and especially useful. Our analysis below (Section 3) considers the chromaticity, CCT, photometry, and relative UV and IR contents of our spectra.

To optimize artificial-vision algorithms for recognizing and identifying colors, researchers require rigorous mathematical analysis of the daylight SPDs that illuminate objects. $^{21,22}$ Yet before our work, suitably large databases of daylight spectra were not readily available. Our collection in fact lets us do a principal-value decomposition (Section 4) that determines the dimensionality of accurate linear models of these spectra.

\section{EXPERIMENTAL PROCEDURE}

Here our SPDs are of hemispheric daylight: global spectral irradiances $E(\lambda)$ on a horizontal surface from direct sunlight (when present) and the entire sky. Over a period of 2 years, we recorded 2600 spectra from the roof of the University of Granada's Science Faculty $\left(37^{\circ} 11^{\prime} \mathrm{N}\right.$, $3^{\circ} 37^{\prime} \mathrm{W}$, altitude $680 \mathrm{~m}$ ), which is in an urban, nonindustrial area. The spectra used here are part of a larger project $^{23}$ and have been used in a variety of ways, including developing an improved algorithm for calculating daylight and skylight CCTs. ${ }^{24,25}$

Our observing site's horizon has few obstructions, and so our spectra sample nearly $2 \pi$ sr of the sky. Specifically, nearby buildings project no more than $9^{\circ}$ above the astronomical horizon and obscure only $\sim 7 \%$ of the sky. Given our detector's cosine response, the effect on our measurements is insignificant. We used a LI-COR model LI-1800 spectroradiometer ${ }^{26}$ equipped with a holographic grating monochromator and a cosine-corrected receptor to measure $E(\lambda)$ from 300 to $1100 \mathrm{~nm}$ in 5 -nm steps (this spectroradiometer is described in more detail elsewhere ${ }^{27-29}$ ). We calibrated the LI-1800 every six months with a 200-W quartz tungsten halogen lamp, and we avoided any strong temperature variations that could change its photodetector's sensitivity.

From February 1996 to February 1998, we made measurements of $E(\lambda)$ in nearly all weather (clear sky, partly cloudy, mostly cloudy, hazy, overcast, misty, etc.), excluding only rainfall and snowfall. We acquired data from sunrise to sunset at 1-h intervals, switching to 10-min intervals when solar-elevation angles $h_{0}<+5^{\circ}$. Our measurements' minimum $h_{0} \sim-4^{\circ}$, because at this and smaller $h_{0}$ the LI-1800 has unacceptably low signal-tonoise ratios. Simultaneously, we used a pyranometer to measure how stable the broadband global irradiance was during a spectral scan: when integrated irradiance varied significantly $(>1 \%)$, we rejected the corresponding $E(\lambda)$ scan.

\section{EXPERIMENTAL MEASUREMENTS}

Figure 1 shows three different daylight spectra measured in Granada, each normalized at $560 \mathrm{~nm}$. The daylight phase whose CCT $=5770 \mathrm{~K}$ corresponds to a clear day with $h_{0}=69^{\circ}$. The curve with CCT $=4250 \mathrm{~K}$ was measured during an overcast sunrise, and the $24,380-\mathrm{K}$ spectrum was taken during a partly cloudy sunrise. Throughout our analysis we calculate CCTs by using the binary search algorithm described in Ref. 25.

\section{A. Chromaticity Coordinates}

Figure 2 shows the CIE 1931 chromaticities of all 2600 Granada daylight spectra overlaid on the Planckian locus and the CIE daylight locus. ${ }^{20}$ Also plotted in Fig. 2 is our Granada daylight locus, which we calculate from a leastsquares fit to be

$$
y=-0.05188+1.55320 x-1.09234 x^{2} .
$$

Our Granada daylight spectra provide a measured chromaticity gamut ${ }^{18}$ unprecedented in the daylight literature. ${ }^{20}$ Most Granada data with low CCTs lie above the Planckian locus and below the CIE locus, in agreement with Judd et al. ${ }^{2}$ However, the two daylight loci differ markedly: Like other researchers, we find that our locus's slope is smaller than that of the CIE locus. $1,3-5,9,10,14$ For high CCTs ( $>9000 \mathrm{~K})$ the CIE locus does not adequately represent Granada daylight chromaticities, which are perceptibly greener than the CIE's. All Granada chromaticity coordinates on the green side of the CIE locus (at CCT $>9000 \mathrm{~K}$ ) are generated by clear skies and small $h_{0}$.

The Granada locus, like the locus derived by Condit and Grum, ${ }^{4}$ is farthest from the Planckian locus at high CCTs (i.e., toward the greens). In fact, the greatest 1931 $x, y$ Euclidean distance between the Granada and CIE daylight loci is $\sim 0.011$, or some seven just-noticeable differences (JNDs). ${ }^{30}$ Our new daylight locus describes its associated chromaticities quite well. In other words, our data scatter very little about it despite the length of our

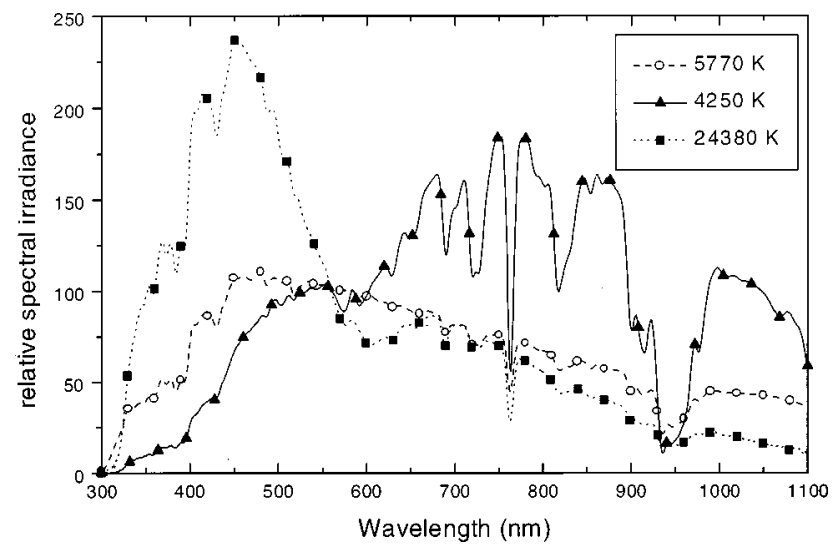

Fig. 1. Relative spectral irradiance, normalized at $560 \mathrm{~nm}$, for three different daylight SPDs measured in Granada. 


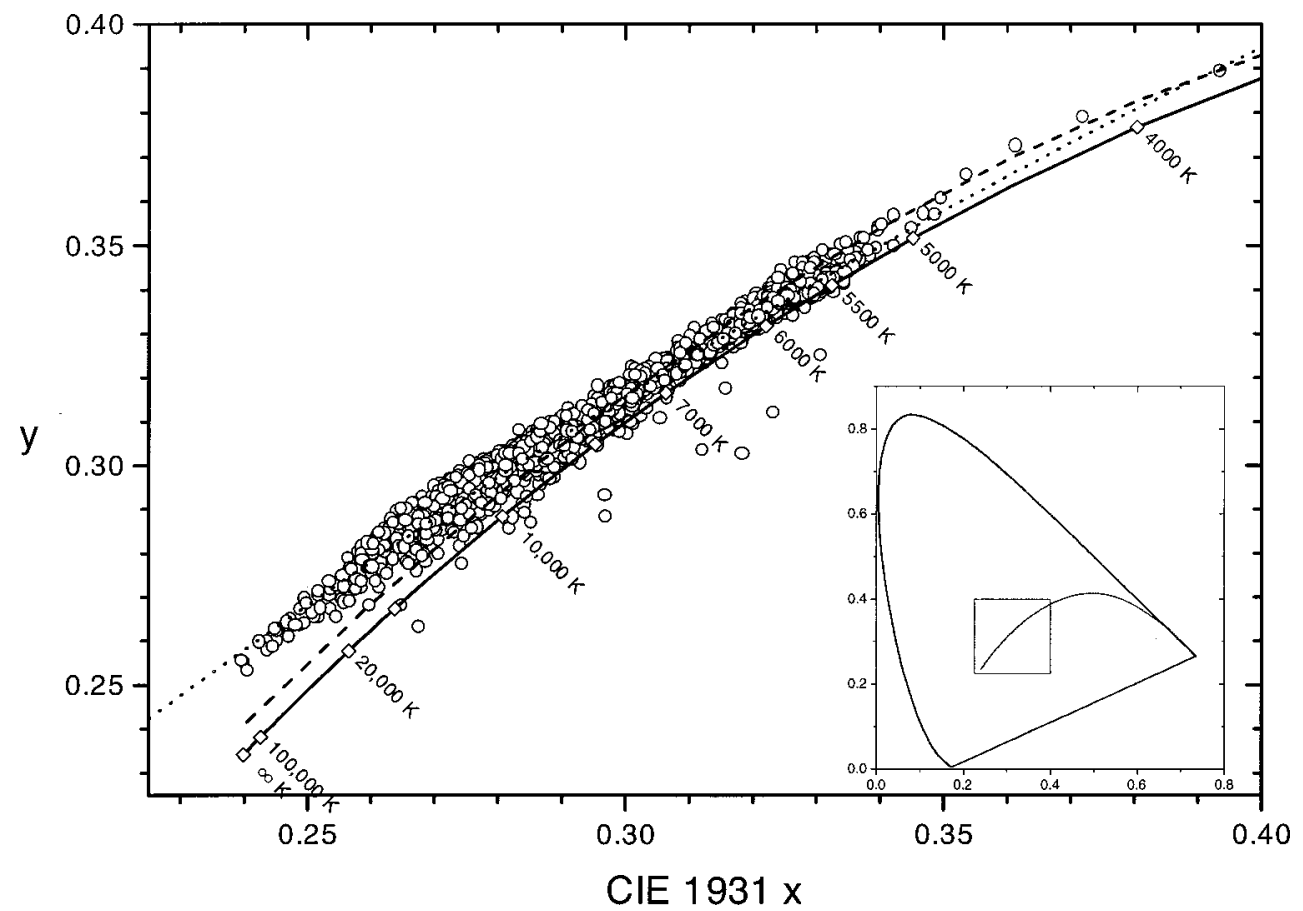

Fig. 2. CIE 1931 chromaticities of our 2600 Granada daylight measurements (circles) overlaid on the CIE daylight locus (dashed curve), the Planckian locus (solid curve with squares), and the Granada daylight locus (dotted curve). The inset shows the entire CIE 1931 diagram and Planckian locus.

measurement campaign and the number of irradiance spectra measured. This small scatter about a daylight locus is comparable to that found by some authors ${ }^{1,3-5,7,10,14}$ but is much less than that observed by others. $^{9,11-13}$ For example, Sastri and Das $^{9}$ did not calculate a daylight locus because their chromaticities spanned so wide an area on the CIE diagram.

Most of our daylight chromaticities are above the Planckian locus toward the greens. In fact, only 30 of our 2600 chromaticities lie below it toward the purples. Although this distribution has often been seen before, ${ }^{1,3,7,10,11}$ some southern-hemisphere researchers find that most of their daylight chromaticities are below the Planckian locus. 9,13,14 To our knowledge, no one has satisfactorily explained this difference between hemispheres, although the wide variety of sites in both suggests that local terrain color is not responsible.

\section{B. Correlated Color Temperatures}

As noted above, researchers have consistently shown that daylight CCTs allow accurate estimates of its visiblewavelength irradiance spectra $E(\lambda)$. This makes CCT a useful shorthand for specifying daylight's colorimetric and spectral characteristics. In fact, the CIE describes the relative spectral irradiances of typical daylight phases at the Earth's surface by invoking CCTs ranging from 4000 to $25,000 \mathrm{~K}^{15}$

The CCT limits for our 2600 daylight measurements are 3758-34,573 K. To make comparing our data with that of other researchers more perceptually meaningful, we use inverse CCT rather than CCT itself. The unit of inverse CCT is the reciprocal mega-Kelvin ( $\left.10^{6} / \mathrm{CCT}\right)$, denoted by the symbol $\mathrm{MK}^{-1}$ (historically known as the "mired"). ${ }^{31}$ Reciprocal mega-Kelvins produce a uniform

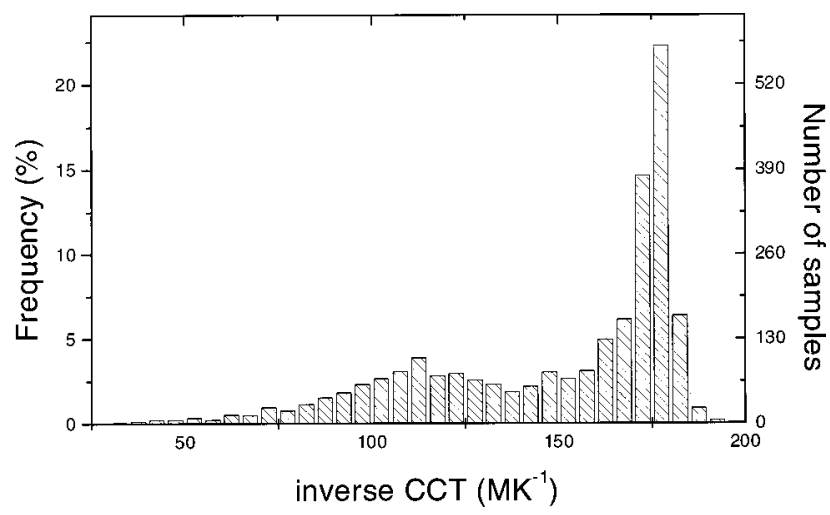

Fig. 3. Histogram of inverse correlated color temperature (CCT) for our 2600 Granada daylight spectra. Each bin is $5 \mathrm{MK}^{-1}$ wide.

scale that better describes human color sensitivity than does CCT proper. On this inverse scale, the inverse CCTs most frequently produced by our Granada daylight spectra range from 175 to $180 \mathrm{MK}^{-1}$ (or 5555 to $5715 \mathrm{~K}$ ), as shown in Fig. 3.

Table 1 compares the most frequently observed (or modal) intervals of inverse CCT from several researchers' daylight measurement campaigns. ${ }^{1,6,7,11,14,20}$ Call this modal interval m(CCT). Only clear-sky hemispheric irradiances have a consistent $\mathrm{m}(\mathrm{CCT})$, and it is $\sim 170-180$ $\mathrm{MK}^{-1}$. Note that we include only a few papers in Table 1 because of (1) intrinsic differences caused by differences in how CCTs were reported and (2) the older practice of collectively analyzing CCTs calculated from spectral radiances and irradiances.

The timing and duration of a researcher's daylight measurements clearly affect the $\mathrm{m}(\mathrm{CCT})$ calculated. As 
Henderson points out, this calculated mode should coincide with that actually existing at the observing site. ${ }^{20}$ In other words, making many measurements of atypical spectra will bias the resulting inverse CCT results. Our long-term, routine acquisition of daylight spectra satisfies Henderson's recommendation, and thus our m(CCT) results are truly representative of most Granada daylight.

Although Table 1 shows that clear daylight CCTs have $\mathrm{m}(\mathrm{CCT}) \sim 170-180 \mathrm{MK}^{-1}$, the CIE in fact recommends using the $\mathrm{D}_{65}$ illuminant with $\mathrm{CCT}=6500 \mathrm{~K}$ (or 154 $\mathrm{MK}^{-1}$ ) as the standard illuminant that closely approximates natural daylight. However, the timing and number of our clear-sky daylight measurements bolster our claim that our $\mathrm{m}(\mathrm{CCT})$ of $175-180 \mathrm{MK}^{-1}$ is indeed the correct one for our site rather than the much smaller $\mathrm{m}(\mathrm{CCT})$ implied by the CIE standard.

To analyze how weather affects daylight chromaticities in Granada, we sorted our 2600 spectra into three bins that represent the sky states listed in Table 2. As it indicates, clear skies occur $\sim 3.5$ times more frequently in Granada than do overcasts. Yet perhaps surprisingly, Fig. 4 shows only subtle differences in inverse-CCT frequency distributions for these two extreme sky states.
Nonetheless, persistent differences do exist: $m(C C T)$ for overcasts is $165-170 \mathrm{MK}^{-1}$, or $10 \mathrm{MK}^{-1}$ smaller (i.e., bluer) than that for clear skies. These results are similar to Dixon's in the southern hemisphere. ${ }^{14}$ We expect this $\mathrm{m}(\mathrm{CCT})$ difference between clear and overcast skies: The former have a higher ratio of yellow sunlight to blue skylight or cloud light than do the latter.

Figure 5 shows the $h_{0}$ dependence of CCT for clear and overcast skies. The mean CCT for overcast skies is greater than that for clear skies, as is its variability (for $h_{0}>10^{\circ}$, the overcasts' mean CCT exceeds that for clear skies at the $5 \%$ significance level). The much more variable nature of an overcast's scattering medium means that sudden, large changes in optical depth can occur, with resulting fluctuations in chromaticity and irradiance beneath the overcast. Figure 5 also shows that neither clear nor overcast skies have truly meaningful average CCTs at small $h_{0}$ (i.e., near and during twilight) because their CCT variance is so large then. Note too that minimum CCT occurs for $20^{\circ}<h_{0}<35^{\circ}$ because direct sunlight most reddens horizontal irradiances then. At still smaller $h_{0}$, both the cosine law and rapidly increasing atmospheric extinction reduce the influence on horizontal

Table 1. Most-Frequent Inverse CCT Intervals m(CCT) as Measured by Various Researchers

\begin{tabular}{|c|c|c|}
\hline Paper & Sky State & $\mathrm{m}(\mathrm{CCT})\left(\right.$ in $\left.\mathrm{MK}^{-1}\right)$ \\
\hline Henderson and & North sky & $165-180$ \\
\hline \multirow[t]{2}{*}{ Hodgkiss $^{1}$} & Total sky, no sun & $145-150$ \\
\hline & Total sky, with sun & $170-175$ \\
\hline Collins $^{6}$ & Skylight from four quadrants, total sky with sun & $165-170$ \\
\hline \multirow[t]{3}{*}{ Winch et $a l . .^{7}$} & South sky & $115-120,160-165$ \\
\hline & Total sky, no sun & $130-135$ \\
\hline & Total sky, with sun & $170-175$ \\
\hline Ando et al. (see & North sky, clear sky & $95-100,120-125$ \\
\hline Henderson ${ }^{20}$ ) & North sky, cloudy, rain or snow & $160-165$ \\
\hline Tarrant $^{11}$ & North sky and south sky & $150-160$ \\
\hline \multirow[t]{5}{*}{ Dixon $^{14}$} & Sunlight plus skylight: & \\
\hline & Partial cloud with direct sun & $177.5-182.5$ \\
\hline & Partial cloud with obscured sun & $162.5-167.5$ \\
\hline & Overcast sky & $167.5-172.5$ \\
\hline & Clear sky & $177.5-182.5$ \\
\hline \multirow[t]{4}{*}{ Present work } & Hemispheric daylight (sunlight when present): & \\
\hline & Clear sky & $175-180$ \\
\hline & Overcast sky & $165-170$ \\
\hline & Few clouds to mostly cloudy (i.e., all others) & $175-180$ \\
\hline
\end{tabular}

Table 2. Classification of our Granada Daylight Spectra by Sky State

\begin{tabular}{lccr}
\hline \multicolumn{1}{c}{ Sky state } & $\begin{array}{c}\text { Number } \\
\text { of Spectra }\end{array}$ & CCT Range & Inverse CCT Range \\
\hline Completely clear & 757 & $5373 \mathrm{~K}-32,754 \mathrm{~K}$ & $30.5 \mathrm{MK}^{-1}-186.1 \mathrm{MK}^{-1}$ \\
Completely overcast & 216 & $3758 \mathrm{~K}-34,572 \mathrm{~K}$ & $28.9 \mathrm{MK}^{-1}-266.1 \mathrm{MK}^{-1}$ \\
Few clouds to mostly cloudy & 1627 & $4864 \mathrm{~K}-26,728 \mathrm{~K}$ & $37.4 \mathrm{MK}^{-1}-205.6 \mathrm{MK}^{-1}$ \\
\hline
\end{tabular}


$60 \quad 65 \quad 70 \quad 75 \quad 80 \quad 8590 \quad 95 \quad 100105110115120125130135140145150155160165170175180185190$

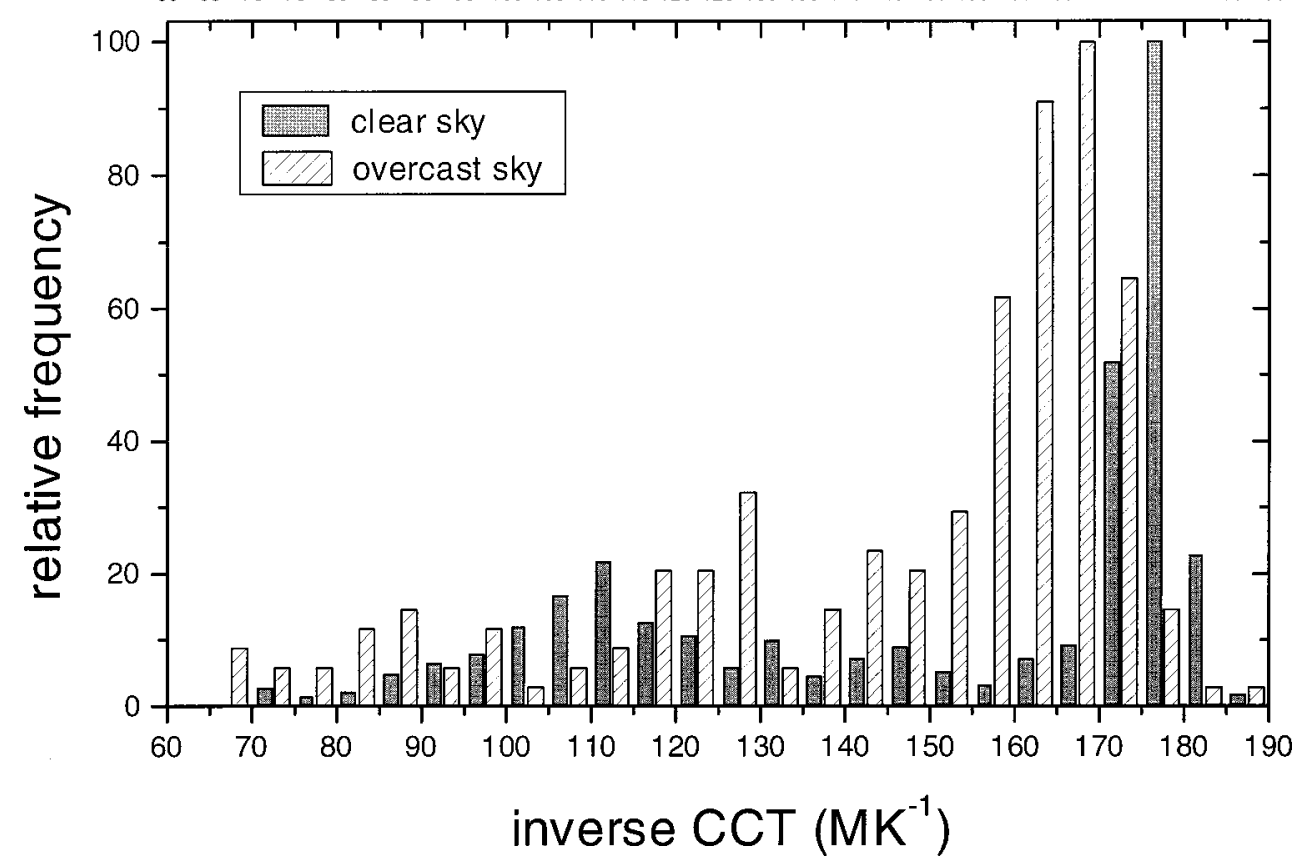

Fig. 4. Histogram of inverse CCT (in $\mathrm{MK}^{-1}$ ) for Granada daylight measured under clear and overcast skies.

$E(\lambda)$ of the direct beam's ever-reddening spectra. At the largest $h_{0}$, the difference between maximum and minimum CCTs is only $\sim 200 \mathrm{~K}$. Still, this difference exceeds 1 JND.

Finally, we looked across an entire year for possible seasonal variations in Granada daylight chromaticities. While a 1-year survey cannot be definitive, we found no significant shifts either in our locus of chromaticities or in the associated histograms of inverse CCT.

\section{Photometric Characteristics}

In many applications, we need photometric rather than radiometric measures of daylight. Because atmospheric scattering and absorption are usually spectrally selective, ideally one calculates illuminance from irradiance rigorously by numerically integrating the spectral convolution of $E(\lambda)$ and the photopic efficiency function $V_{p}(\lambda){ }^{32}$ A less involved (and less robust) technique is to use a single number that relates illuminance to integrated irradiance. This number, the luminous efficacy factor, is the ratio of corresponding photometric and radiometric quantities for a particular spectrum (here, the ratio of illuminance to irradiance integrated over the solar spectrum). Such a factor lets us convert, say, a pyranometer's irradiances to illuminances.

Given our spectroradiometer's spectral range, we integrate $E(\lambda)$ from 300 to $1100 \mathrm{~nm}$ when calculating luminous efficacy factors. Restricting this integration to the visible $(380-780 \mathrm{~nm})$ will yield significantly larger factors, since by definition $V_{p}(\lambda)=0$ outside the visible. Note too that the spectrum-dependent luminous efficacy factor differs from the constant value of the maximum photopic luminous efficacy, $K_{m}=683 \mathrm{lumen} / \mathrm{W} .{ }^{32}$ As convenient as luminous efficacy factors are, using a variable radiometric-to-photometric conversion clearly poses potential pitfalls. Perez et al. note that the relationship

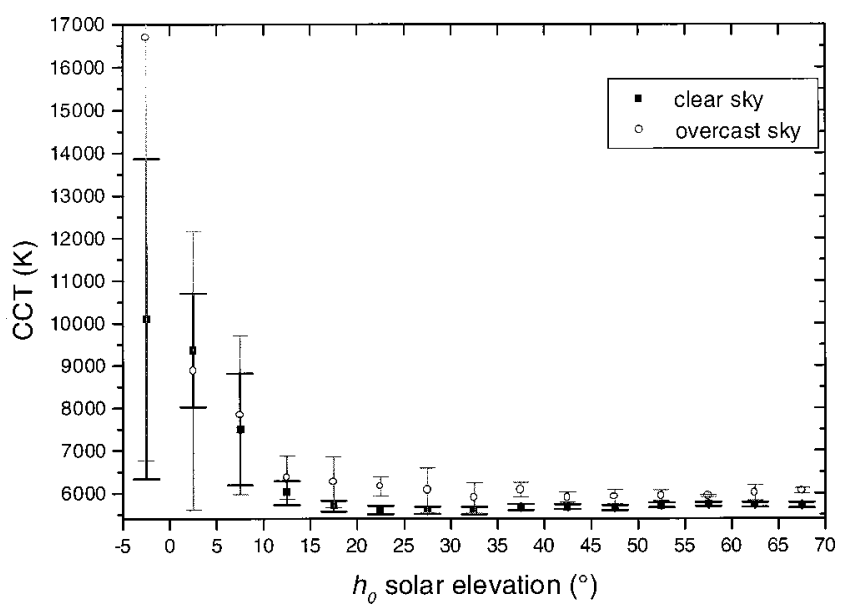

Fig. 5. CCT versus solar elevation $h_{0}$ at $5^{\circ}$ intervals for clear and overcast skies. Squares and circles indicate mean CCTs; each error bar spans 2 standard deviations.

between analogous radiometric and photometric quantities "still leaves many questions unanswered, causing potentially large errors when converting one type of quantity into the other..." (Ref. 33, p. 33).

Researchers often use broadband instruments instead of spectroradiometers to measure outdoor illumination. ${ }^{33-37}$ Broadband devices may have filters whose spectral transmissivity, when convolved optically with the detector's spectral sensitivity, yields a net spectral response that closely resembles $V_{p}(\lambda)$. However, because no broadband filter-detector combination matches $V_{p}(\lambda)$ exactly, it is useful to develop a database of daylight illuminances that are rigorously calculated under many different conditions. For a given daylight phase, this database lets a researcher accurately convert 
broadband irradiance to illuminance or correct a photometer's fixed illuminance conversion.

Figure 6 shows such a database of luminous efficacy

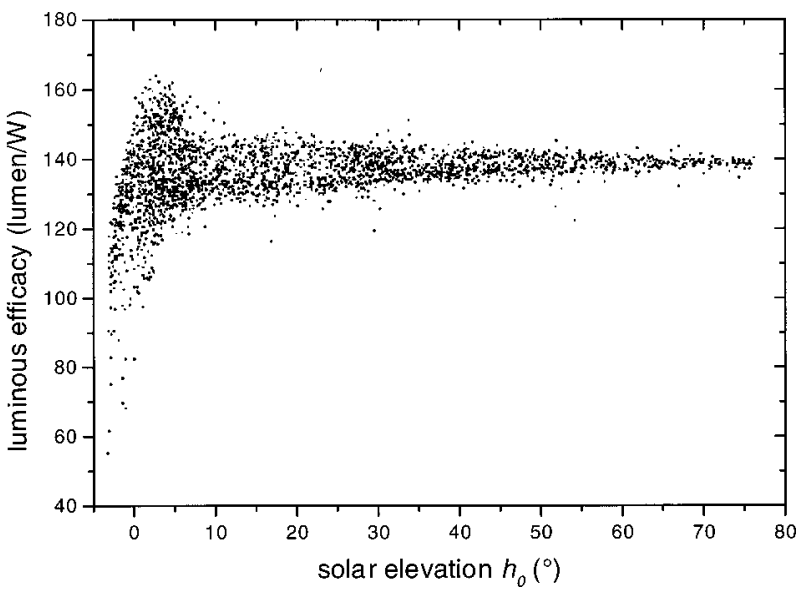

Fig. 6. Photopic luminous efficacies [based on $300-1100 \mathrm{~nm}$ $E(\lambda)$ integrals] as a function of $h_{0}$ for our 2600 Granada daylight spectra.

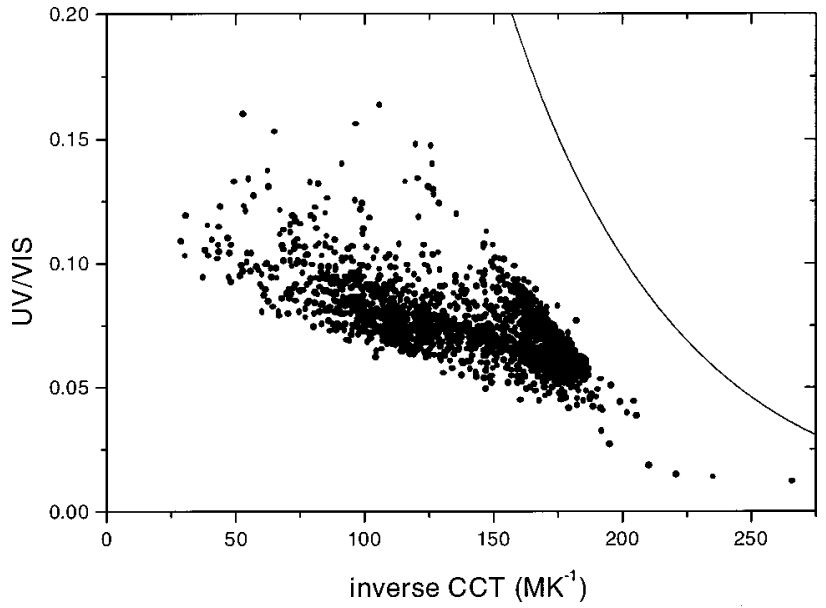

Fig. 7. Ratios of ultraviolet $(300-380 \mathrm{~nm})$ to visible $(380-780$ $\mathrm{nm}$ ) irradiance as a function of inverse CCT (in $\mathrm{MK}^{-1}$ ). The solid curve shows these ratios for Planckian radiators with corresponding color temperatures.

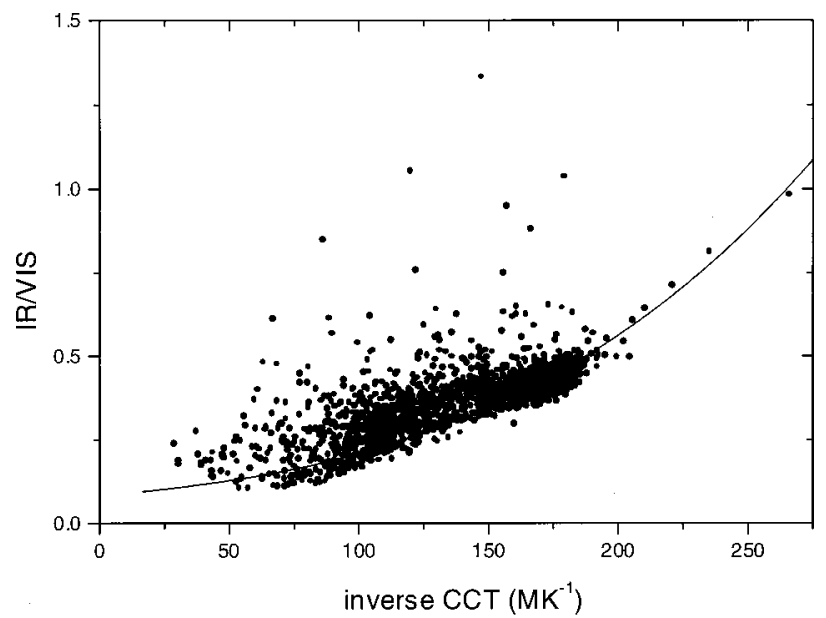

Fig. 8. Ratios of near-infrared $(780-1100 \mathrm{~nm})$ to visible (380$780 \mathrm{~nm}$ ) irradiance as a function of inverse CCT (in $\mathrm{MK}^{-1}$ ). The solid curve shows these ratios for Planckian radiators with corresponding color temperatures. factors calculated from our Granada daylight spectra. These factors are functions of $h_{0}$ because $E(\lambda)$ 's shape changes with $h_{0}$, meaning that even identical integrated irradiances may produce different luminous efficacies. For $h_{0}>20^{\circ}$, luminous efficacy is nearly constant at $\sim 140$ lumen/W. However, it increases with decreasing $h_{0}$, reaching maximum values (and maximum variability) when the sun is near the horizon $\left(h_{0} \sim 5^{\circ}\right)$ and the shapes of $E(\lambda)$ and $V_{p}(\lambda)$ are most similar. Luminous efficacy plummets after sunset as twilight $E(\lambda)$ get progressively bluer [i.e., they are poorer spectral matches to $\left.V_{p}(\lambda)\right]$.

\section{UV and IR Content}

Because daylight's ultraviolet component can profoundly affect the human body, being able to infer it accurately from visible-wavelength irradiances is quite useful. However, Fig. 7 shows that such inferences are problematic at best. In it, for each Granada daylight spectrum we calculate the ratio of ultraviolet $(300-380 \mathrm{~nm})$ to visible (VIS) (380-780 nm) irradiances and then plot these UV/VIS ratios versus inverse CCT for the visible $E(\lambda)$. We also include in Fig. 7 as a reference locus the UV/VIS ratios for Planckian radiators that have the same range of color temperatures as our measured $E(\lambda)$. At all CCTs our Granada ratios are both smaller and more weakly dependent on inverse CCT than those reported by Henderson and Hodgkiss ${ }^{1}$ and Winch et al. ${ }^{7}$ Yet our UV/VIS ratios do resemble those of Judd et $a l^{2}{ }^{2}$ at low, although not high, inverse CCTs.

Near-infrared daylight has attracted less attention from researchers than the UV (in fact, apparently only Dixon discusses it). ${ }^{14}$ Historically this neglect stems from the limited (or nonexistent) IR irradiances in most daylight measurements. To redress this shortcoming, we calculate the ratio of infrared $(780-1100 \mathrm{~nm})$ to visible $(380-780 \mathrm{~nm})$ irradiances for all 2600 of our Granada daylight spectra. As shown in Figure 8, these IR/VIS ratios' correlation with inverse CCT is much larger than that for the UV/VIS ratios. In fact, as Dixon points out ${ }^{14}$ and Fig. 8 reiterates, the IR/VIS ratios' relationship to inverse CCT strongly resembles that for Planckian radiators.

Although the UV/VIS and IR/VIS ratios in Figs. 7 and 8 differ from some reported elsewhere, ${ }^{1,2,7,14}$ this does not necessarily mean that the UV or IR content of Granada daylight is unusual. Our UV/VIS and IR/VIS ratios tell us only about the relative balance between Granada's visible and invisible daylight components, and this balance depends strongly on the quantity measured (daylight or skylight) and on prevailing atmospheric conditions. For example, compared with skylight spectra, our daylight spectra usually include direct sunlight and so will have proportionally less UV energy. Conversely, our spectra's relative IR content will be enhanced whenever they include direct sunlight.

\section{SPECTRAL ANALYSIS}

Color analysis and synthesis often use linear models to represent illuminants. When paired with similar reflec- 
tance models, these permit the design of efficient artificial-vision algorithms. ${ }^{21,22}$ In order to achieve such efficiencies, we need to determine the smallest dimension for our linear daylight models that lets us reconstruct the original $E(\lambda)$ to within some specified accuracy. To determine this minimum dimensionality, we perform a

Table 3. Spectral Regions of Our 2600 Granada Daylight Spectra ${ }^{a}$

\begin{tabular}{lc}
\hline $\begin{array}{c}\text { Spectral Region } \\
(\mathrm{nm})\end{array}$ & $\begin{array}{c}\text { Spectral Irradiances } \\
\text { per Region }\end{array}$ \\
\hline $300-380$ & 17 \\
$780-1100$ & 65 \\
$380-780$ & 81 \\
$300-1100$ & 161 \\
\hline
\end{tabular}

${ }^{a}$ Shown are the number of spectral irradiances in each region for our spectroradiometer's 5-nm resolution. principal-value decomposition of our 2600 Granada daylight spectra.

We begin by calculating how many 5 -nm-wide spectral bins exist in each of the spectral regions of interest for analyzing daylight: the UV, visible, IR, and their combination (Table 3). Given the variability of daylight in these spectral regions, 5-nm spectral resolution yields more-reliable reconstructions than does 10-nm resolution. $^{24}$ Next we determine the eigenvectors needed to reconstruct a given set of spectra as described by Parkinnen et $a l .{ }^{38}$ If $E_{E}(\lambda)$ is a measured daylight spectrum, then we can approximate it by using $p$ eigenvectors in

$$
E_{R}(\lambda)=\sum_{i=1}^{p}\left\langle E_{E}(\lambda) \mid V_{i}(\lambda)\right\rangle V_{i}(\lambda),
$$

where $E_{R}(\lambda)$ is the reconstructed spectrum, $V_{i}(\lambda)$ is the $i$ th eigenvector, and $\langle\mid\rangle$ denotes the inner product. To re-

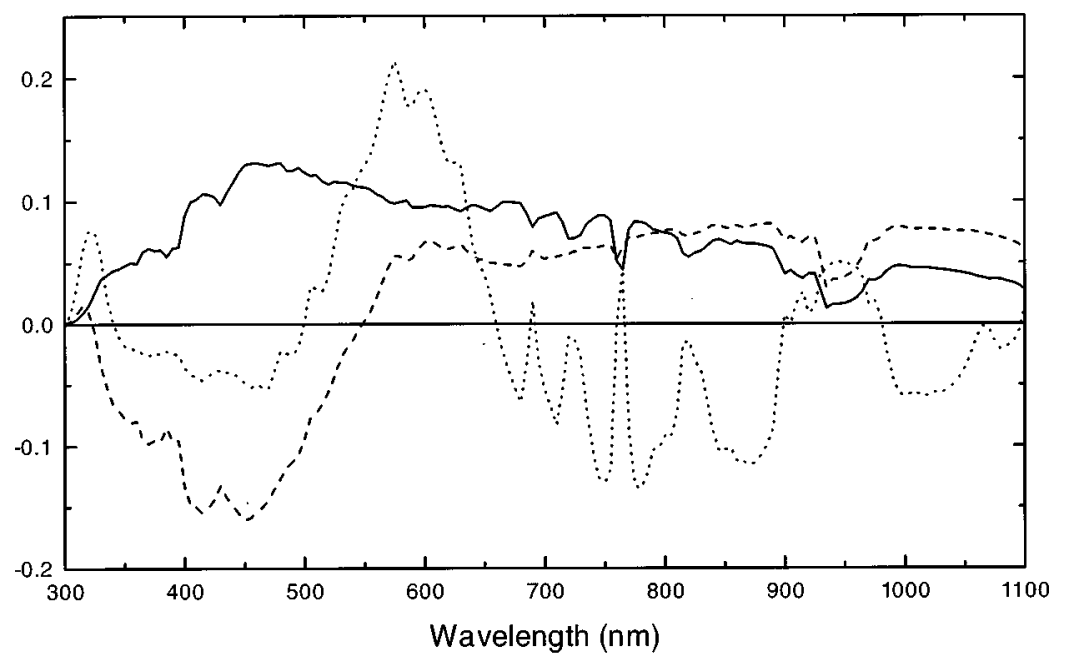

(a)

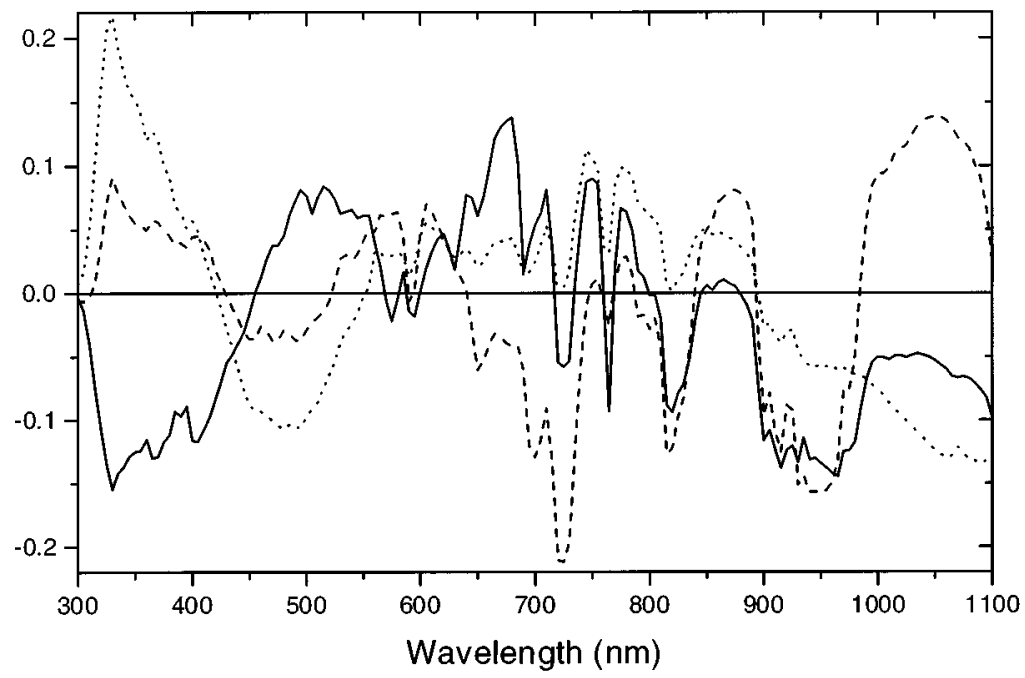

(b)

Fig. 9. (a) Spectral distribution from 300-1100 nm of eigenvectors $V_{1}(\lambda), V_{2}(\lambda)$, and $V_{3}(\lambda)$ for our Granada daylight basis set. Solid curve, $i=1$ eigenvector $V_{1}(\lambda)$ [the mean $E(\lambda)$ ]; dashed curve, $i=2$ eigenvector $V_{2}(\lambda)$; dotted curve, $i=3$ eigenvector $V_{3}(\lambda)$. (b) Spectral distribution from 300 to $1100 \mathrm{~nm}$ of eigenvectors $V_{4}(\lambda), V_{5}(\lambda)$, and $V_{6}(\lambda)$ for our Granada daylight basis set. Solid curve, $i$ $=4$ eigenvector $V_{4}(\lambda)$; dashed curve, $i=5$ eigenvector $V_{5}(\lambda)$; dotted curve, $i=6$ eigenvector $V_{6}(\lambda)$. 
construct a curve of $N$ points exactly, in principle we need $N$ eigenvectors. In practice, however, correlations among our 2600 daylight spectra mean that we can set $p \ll N$ without losing any meaningful spectral information.

In order to reconstruct SPDs with any desired accuracy, we must adopt the approach of Eq. (2) rather than use the approximate CIE technique. ${ }^{15}$ Only the former lets us adequately analyze the dimensionality $p$ needed to achieve a given accuracy of reconstruction. To test our reconstructed daylight spectra, we once again use a goodness-of-fit coefficient ${ }^{17,23,24}$ (GFC) that is based on the inequality of Schwartz. Here this coefficient is

$$
\mathrm{GFC}=\frac{\left|\Sigma_{j} E_{E}\left(\lambda_{j}\right) E_{R}\left(\lambda_{j}\right)\right|}{\left|\Sigma_{j}\left[E_{E}\left(\lambda_{j}\right)\right]^{2}\right|^{1 / 2}\left|\Sigma_{j}\left[E_{R}\left(\lambda_{j}\right)\right]^{2}\right|^{1 / 2}} .
$$

GFC is the multiple correlation coefficient $R$, the square root of $E_{R}(\lambda)$ 's spectral variance with respect to the original $E_{E}(\lambda)$. GFC ranges from 0 to 1 , where 1 indicates a perfect reconstruction. We found that colorimetrically accurate $E_{R}(\lambda)$ require $\mathrm{GFC} \geqslant 0.995$; what we call "good" spectral fit requires $\mathrm{GFC} \geqslant 0.999$, and $\mathrm{GFC} \geqslant 0.9999$ is necessary for "excellent" or almost-exact fit. Examples of $E_{E}(\lambda)$ with different GFCs are found in Refs. 17 and 24.

In Fig. 9 we plot the first six Granada daylight $V_{i}(\lambda)$ for $300-1100 \mathrm{~nm}$. The $i=1$ eigenvector $V_{1}(\lambda)$ corresponds to our database's mean $E(\lambda)$, and its spectral details include only a few small absorption bands. As Judd et $a l{ }^{2}$ and Sastri and $\operatorname{Das}^{9}$ note, the $V_{2}(\lambda)$ eigenvector accounts for most yellow-blue chromaticity variations. Like the mean $E(\lambda), V_{2}(\lambda)$ also varies smoothly, increasing from negative to positive values near $\lambda=550 \mathrm{~nm}$. Starkly different is the $V_{3}(\lambda)$ eigenvector, many of whose sharp peaks derive from atmospheric absorption bands. Here we part company with Judd et al. and Sastri and
Das: Unlike them, we find no purple-green shift associated with $V_{3}(\lambda)$. As Fig. 10 shows, $V_{3}(\lambda)$ actually yields chromaticity shifts nearly parallel to those of $V_{2}(\lambda)$. To determine the chromaticity shifts plotted in Fig. 10, we calculate the chromaticity pairs that result when we add and subtract either $V_{2}(\lambda)$ or $V_{3}(\lambda)$ to and from $V_{1}(\lambda)$.

Although our collection of daylight spectra is the largest measured to date, we require only a few $V_{i}(\lambda)$ to account for nearly all its variance in the visible. Unlike earlier researchers, ${ }^{4,9}$ we find that the first five $V_{i}(\lambda)$ account for $99.991 \%$ of the observed variance between 380 and $780 \mathrm{~nm}$. This good performance in the visible is not

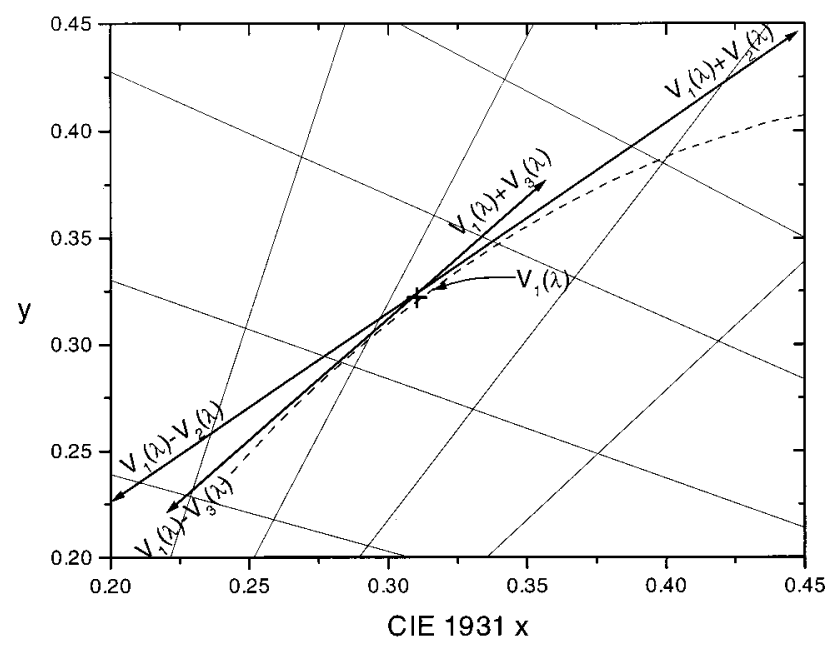

Fig. 10. Colorimetric characteristics of the eigenvectors shown in Fig. 10(a), with the chromaticity of the mean vector $V_{1}(\lambda)$ marked "+." For $V_{2}(\lambda)$ and $V_{3}(\lambda)$ we plot the chromaticity shift that each accounts for (lines with arrows). We also include the deuteranopic and tritanopic confusion lines (skewed grid lines) as well as the Planckian (dashed curve).

Table 4. Percentage of Reconstructed Spectra $E_{R}(\lambda)$ That Exceed Our Three Target GFC Values As a Function of Spectral Region and Number of Eigenvectors $p$ Used in Eq. (2) (Here, $2 \leqslant p \leqslant 8)^{a}$

\begin{tabular}{|c|c|c|c|c|c|c|c|}
\hline \multirow{2}{*}{$\begin{array}{c}\text { Spectral } \\
\text { Region (nm) } \\
\text { [Number of } \\
E(\lambda) \\
\text { per spectrum] }\end{array}$} & \multicolumn{7}{|c|}{$p$ Eigenvectors } \\
\hline & 2 & 3 & 4 & 5 & 6 & 7 & 8 \\
\hline $300-380$ & 99.7 & 99.9 & 100 & & & & \\
\hline \multirow[t]{2}{*}{ (17) } & 95.9 & 98.0 & 98.5 & 98.7 & 98.8 & 99.3 & 99.4 \\
\hline & 37.4 & 78.6 & 93.4 & 93.6 & 94.5 & 94.9 & 95.3 \\
\hline $780-1100$ & 90.0 & 99.8 & 100 & & & & \\
\hline \multirow[t]{2}{*}{ (65) } & 54.6 & 98.0 & 99.3 & 99.5 & 99.8 & 99.8 & 99.8 \\
\hline & 8.4 & 43.0 & 80.5 & 93.0 & 95.3 & 97.2 & 97.4 \\
\hline $380-780$ & 95.2 & 99.8 & 100 & & & & \\
\hline \multirow[t]{2}{*}{$(81)$} & 78.0 & 97.4 & 99.5 & 99.9 & 100 & & \\
\hline & 3.8 & 29.3 & 61.5 & 91.5 & 97.2 & 99.1 & 99.4 \\
\hline $300-1100$ & 92.5 & 98.3 & 99.5 & 99.9 & 100 & & \\
\hline \multirow[t]{2}{*}{$(161)$} & 37.9 & 70.7 & 88.5 & 97.1 & 99.6 & 99.8 & 99.8 \\
\hline & 0.3 & 4.5 & 17.5 & 48.7 & 70.7 & 88.0 & 92.3 \\
\hline
\end{tabular}

${ }^{a}$ Within each cell, rows from top to bottom are for target $\mathrm{GFC}=0.995,0.999$, and 0.9999. 
surprising given the Granada chromaticities' close proximity to their daylight locus (Fig. 2). Elsewhere in the spectrum, however, the $E(\lambda)$ correlation is not as high. For example, the correlation is lower from 300 to $380 \mathrm{~nm}$ (which includes UVA and UVB), although reduced accuracy of our spectroradiometer here may increase variance. As in the visible, from 300 to $380 \mathrm{~nm}$ the first five $V_{i}(\lambda)$ account for $99.990 \%$ of the total observed variance, even though the visible spans a wavelength region five times larger (and thus $p=5$ might be expected to account for less variance there). In the LI-1800's $320-\mathrm{nm}$ wide IR band, the first five $V_{i}(\lambda)$ account for about as much of the total variance as they do in the UV.

Next we use the CIE method ${ }^{15}$ to estimate daylight $E_{E}(\lambda)$ from their associated chromaticities. However, we update this method by using the Granada $V_{i}(\lambda)$ shown in Fig. 9(a) and Eq. (1)'s Granada daylight locus. Because we reconstruct $E_{R}(\lambda)$ using $p=3$, the mean GFC obtained for our $2600 E_{R}(\lambda)$ is only 0.99543 . Moreover, we note that merely $33 \%$ of these $E_{R}(\lambda)$ have GFC $>0.999$ and only $4.6 \%$ have GFC $>0.9999$.

Using $2 \leqslant p \leqslant 8$ in Eq. (2), we calculated all 2600 $E_{R}(\lambda)$ for Table 3's four spectral ranges. Table 4 and Fig. 11 indicate the accuracies possible for these upper limits. Not surprisingly, mean GFC increases with $p$, although the increase is not uniform in all spectral regions. In particular, with $p=2$ the UV yields the highest mean GFC compared with the remaining spectral regions. By contrast, using $p>5$ scarcely increases mean GFC in the UV from its $p=4$ values.

Figure 11 and Table 4 indicate that the visible produces the best mean GFC and that the greatest variability (smallest correlation) exists in the UV and IR. For the mean GFC in all four spectral ranges to be considered "good," we require $p \geqslant 3$, and for it to be "almost exact" we need $p \geqslant 6$. However, we should not choose $p$ solely on the basis of mean GFC, independent of spectral region. Instead, we need to see how few $V_{i}(\lambda)$ are required for us to meet our target GFCs in each spectral region (Table 4). For $95 \%$ of $E_{R}(\lambda)$ to have at least "good" quality, the minimum $p$ varies from 2 in the narrowest region (the UV) to 5 in the widest (300-1100 nm). For $95 \%$ of $E_{R}(\lambda)$ to be "almost exact," $p$ may need to be as high as 10 from 300 to $1100 \mathrm{~nm}, 8$ in the UV, or 6 in the visible and IR.

The CIE recommends using only three basis vectors (although with 2 degrees of freedom each) to achieve a univocal relationship between chromaticities and reconstructed $E(\lambda)$. Yet if we want a spectrally "almost-exact" $E_{R}(\lambda)$ at visible wavelengths, we must use $p \geqslant 6$ in Eq. (2). Adding either the UV or the IR region to the visible requires $p \geqslant 7$ (as Slater and Healey note). ${ }^{16}$ If we want highly accurate $E_{R}(\lambda)$ from 300 to $1100 \mathrm{~nm}$, we must use $p=10$.

Although increasing $p$ always improves $E_{R}(\lambda)$ 's average spectral quality, note that this extra computational burden soon produces only marginal colorimetric improvements in $E_{R}(\lambda)$. Because the relationship between $E_{R}(\lambda)$ 's spectral and colorimetric accuracy is not simple, we perform yet another test. In order to evaluate the size of color differences, we convert our colorimetric data to the CIELUV uniform color space. ${ }^{39}$ In calculating the CIELUV color difference $\Delta E_{u v}^{*}$ we use ${ }^{40}$

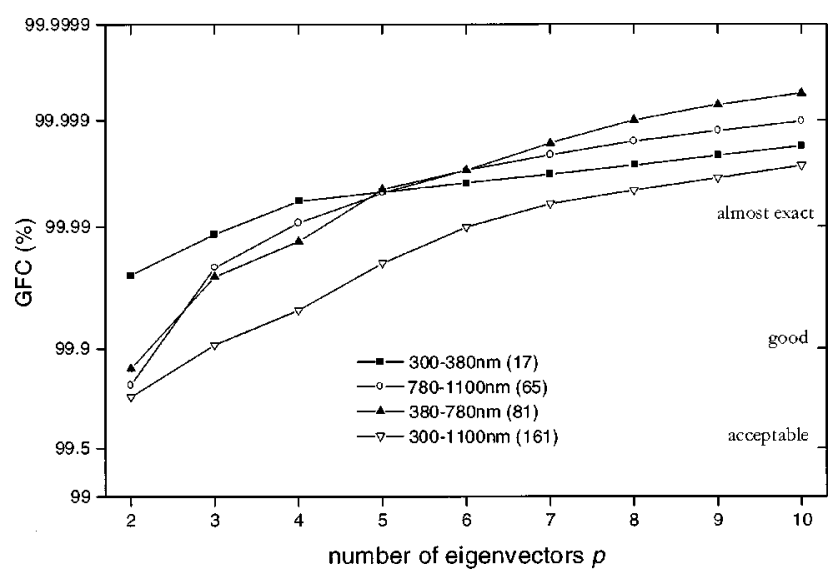

Fig. 11. Mean GFC for our 2600 Granada daylight spectra, with use of $2 \leqslant p \leqslant 10$ eigenvectors in Eq. (2) for the indicated spectral region. We label in parentheses the number of spectral irradiances within each region for 5 -nm resolution.

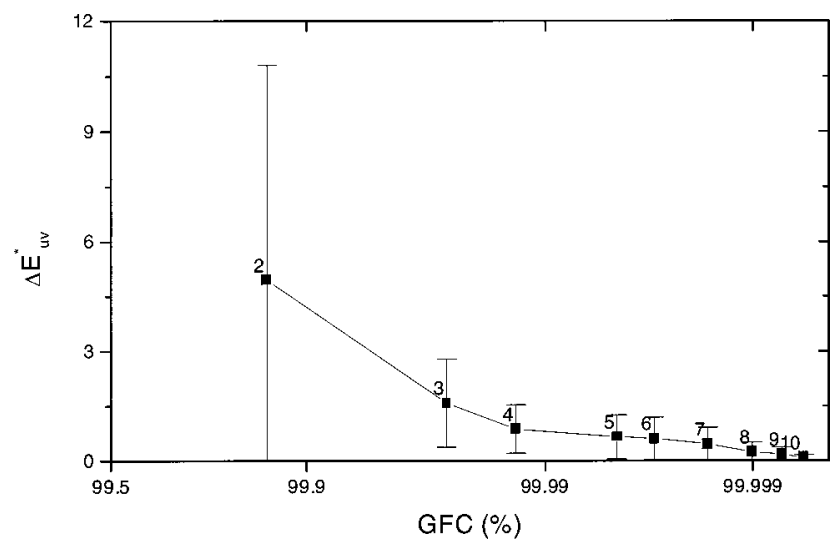

Fig. 12. Mean CIELUV color difference $\Delta E_{u v}^{*}$ (Eq. 4) as a function of mean GFC. Numbers next to the squares indicate the number of eigenvectors $p$ needed in Eq. (2) to obtain the given GFC. Each error bar spans 2 standard deviations.

$$
\Delta E_{u v}^{*}=\left[\left(\Delta L^{*}\right)^{2}+\left(\Delta u^{*}\right)^{2}+\left(\Delta v^{*}\right)^{2}\right]^{1 / 2},
$$

where $L^{*}, u^{*}$, and $v^{*}$ are the CIELUV space's orthogonal coordinates, and $\Delta L^{*}, \Delta u^{*}, \Delta v^{*}$ are the corresponding differences between coordinates of the original and the reconstructed colors.

Figure 12 shows how the mean CIELUV color difference between $E_{E}(\lambda)$ and $E_{R}(\lambda)$ changes as $p$ increases from 2 to 10 (the mean here is over all 2600 daylight spectra). As $p$ increases, the mean colorimetric distance $\Delta E_{u v}^{*}$ decreases sharply, especially for $p<5$. Three or five $\Delta E_{u v}^{*}$ units are often taken to be 1 JND in technical and industrial applications. Thus using $p=3$ in Eq. (2) means that both the mean $\Delta E_{u v}^{*}$ and its standard deviation will satisfy this practical JND criterion. So from a colorimetric (rather than a spectral) standpoint, the CIE's recommendation of three basis vectors is adequate. Note that we reached this conclusion by having the original $E_{E}(\lambda)$ available to use in Eq. (2). Yet when we actually implement color identification and recognition algorithms, we will lack the original $E_{E}(\lambda)$ and instead must rely on sensors with poorer spectral resolution and perhaps additional noise. Thus for truly reliable results, 
prudence suggests that we use more than the absolute minimum number of $V_{i}(\lambda)$ in Eq. (2), with $p=4$ striking a good compromise between efficiency and error.

\section{CONCLUSIONS}

Our suite of horizontal daylight $E(\lambda)$ provides an exceptionally broad range of natural-light chromaticities and CCTs. The differences between the CIE and the Granada daylight loci are most evident at high CCTs (>9000 K), where the Granada locus is greener. If we had to recommend one CCT as best characterizing Granada daylight, our analysis suggests $5700 \mathrm{~K}$. Our measurements also indicate that Granada's mostfrequent inverse CCT [m(CCT)] under clear skies is 175$180 \mathrm{MK}^{-1}$ (or $5555-5715 \mathrm{~K}$ ), a value that decreases to 165-170 $\mathrm{MK}^{-1}$ (or 5882-6060 K) under overcasts. Granada's UV/VIS ratios are less dependent on CCT than any reported previously; the analogous IR/VIS ratios nearly duplicate those of Planckian radiators with the same color temperatures.

A principal-components analysis of our 2600 Granada daylight spectra indicates that the $i=2$ eigenvector accounts for their yellow-blue chromaticity variations and that the $i=3$ eigenvector does not yield any purplegreen variation, although it does depend on atmospheric absorption bands. Spectrally speaking, our work shows that we need $p>5$ for accurate $E_{R}(\lambda)$ in the visible, even though CIE recommendations for reconstructing daylight SPDs from chromaticities in effect call only for $p=3$. If we include either the UV or the IR in our $E_{R}(\lambda)$, the minimum $p$ increases to 7 ; if we want to construct the entire 300-1100 nm spectrum, we need 10 vectors. As the CIE recommends, $p=3$ will produce $E_{R}(\lambda)$ that are colorimetrically indistinguishable from the corresponding $E_{E}(\lambda)$ for most observers, although in machine-vision applications a modest increase to $p=4$ seems advisable.

We would not be surprised if subsequent daylight measurement campaigns in other countries produce colorimetric and spectral analyses that differ perceptibly from ours. However, our earlier work suggests that daylight spectra do share some fundamental, quantifiable similarities worldwide. ${ }^{24}$ Thus we view this paper as a vital first step in determining whether and to what extent sitedependent differences are significant in daylight. More immediately, our analysis of the largest extant set of hemispheric daylight spectra offers useful baseline information for any site that lacks comparable data. As is true elsewhere in atmospheric optics, revisiting the welltrod ground of daylight spectra still affords fresh spectral and colorimetric insights. In addition to providing a large, valuable library of such data, we believe that our work suggests new ways of understanding and solving some quite practical problems associated with outdoor vision, whether machine or human.

\section{ACKNOWLEDGMENTS}

J. Hernández-Andrés, J. Romero, and J. Nieves were supported by Spain's Comisión Interministerial de Ciencia y Tecnología (CICYT) under research grant PB96-1454. R. L. Lee was supported by U.S. National Science Founda- tion grant ATM-9820729 and by the U.S. Naval Academy's Departments of Mathematics and Physics.

Corresponding author Javier Hernández-Andrés can be reached at the address on the title page or by e-mail, javierha@ugr.es.

\section{REFERENCES AND NOTES}

1. S. T. Henderson and D. Hodgkiss, "The spectral energy distribution of daylight," Br. J. Appl. Phys. 14, 125-131 (1963).

2. D. B. Judd, D. L. MacAdam, and G. Wyszecki, "Spectral distribution of typical daylight as a function of correlated colour temperature," J. Opt. Soc. Am. 54, 1031-1041 (1964).

3. G. J. Chamberlin, A. Lawrence, and A. A. Belbin, "Observations on the related colour temperature of north daylight in southern England," Light Light. 56, 70-72 (1963).

4. H. R. Condit and F. Grum, "Spectral energy distribution of daylight," J. Opt. Soc. Am. 54, 937-944 (1964).

5. Y. Nayatani and G. Wyszecki, "Color of daylight from north sky,” J. Opt. Soc. Am. 53, 626-629 (1963).

6. J. F. Collins, "The colour temperature of daylight," Br. J. Appl. Phys. 16, 527-532 (1965).

7. G. T. Winch, M. C. Boshoff, C. J. Kok, and A. G. du Toit, "Spectroradiometric and colorimetric characteristics of daylight in the southern hemisphere: Pretoria, South Africa," J. Opt. Soc. Am. 56, 456-464 (1966).

8. S. R. Das and V. D. P. Sastri, "Spectral distribution and color of tropical daylight," J. Opt. Soc. Am. 55, 319-323 (1965).

9. V. D. P. Sastri and S. R. Das, "Typical spectral distributions and colour for tropical daylight," J. Opt. Soc. Am. 58, 391398 (1968).

10. Y. Nayatani, M. Hitani, and H. Minato, "Chromaticity and spectral energy distribution of daylight from north sky at Amagasaki, Japan,” Bull Electrotech. Lab. 31, 1127-1135 (1967)

11. A. W. S. Tarrant, "The spectral power distribution of daylight," Trans. Illum. Eng. Soc. 33, 75-82 (1968).

12. G. L. Knestrick and J. A. Curcio, "Measurements of the spectral radiance of the horizon sky," Naval Research Laboratory Rep. 6615 (Naval Research Laboratory, Washington, D.C., 1967).

13. V. D. P. Sastri and S. B. Manamohanan, "Spectral distribution and colour of north sky at Bombay," J. Phys. D Appl. Phys. 4, 381-386 (1971)

14. E. R. Dixon, "Spectral distribution of Australian daylight," J. Opt. Soc. Am. 68, 437-450 (1978).

15. G. Wyszecki and W. S. Stiles, Color Science: Concepts and Methods, Quantitative Data and Formulae (Wiley, New York, 1982), pp. 144-146.

16. D. Slater and G. Healey, "Analyzing the spectral dimensionality of outdoor visible and near-infrared illumination functions," J. Opt. Soc. Am. A 15, 2913-2920 (1998).

17. J. Romero, A. García-Beltrán, and J. Hernández-Andrés, "Linear bases for representation of natural and artificial illuminants," J. Opt. Soc. Am. A 14, 1007-1014 (1997).

18. R. L. Lee, Jr., "Twilight and daytime colors of the clear sky,” Appl. Opt. 33, 4629-4638, 4959 (1994).

19. See Ref. 15, p. 11.

20. S. T. Henderson, Daylight and Its Spectrum (American Elsevier, New York, 1970).

21. L. T. Maloney and B. A. Wandell, "Color constancy: a method for recovering surface spectral reflectance," J. Opt. Soc. Am. A 3, 29-33 (1986).

22. D. H. Marimont and B. A. Wandell, "Linear models of surface and illuminant spectra," J. Opt. Soc. Am. A 9, 19051913 (1992).

23. J. Hernández-Andrés, "Características espectrales y colorimétricas de la luz-día y luz-cielo en Granada," Ph.D. dissertation (Universidad de Granada, Granada, Spain, 1999).

24. J. Hernández-Andrés, J. Romero, A. García-Beltrán, and J. L. Nieves, "Testing linear models on spectral daylight measurements," Appl. Opt. 37, 971-977 (1998). 
25. J. Hernández-Andrés, R. L. Lee, Jr., and J. Romero, "Calculating correlated color temperatures across the entire gamut of daylight and skylight chromaticities," Appl. Opt. 38, 5703-5709 (1999).

26. LI-1800 spectroradiometer from LI-COR, Inc., 4421 Superior Street, Lincoln, Nebraska 68504-1327.

27. C. Riordan, D. R. Myers, R. Hulstrom, W. Marion, C. Jennings, and C. Whitaker, "Spectral solar radiation data base at SERI," Sol. Energy 20, 67-79 (1989).

28. D. R. Myers, "Estimates of uncertainty for measured spectra in the SERI spectral solar radiation database," Sol. Energy 43, 347-353 (1989).

29. S. Nann and C. Riordan, "Solar spectral irradiance under clear and cloudy skies: measurement and semiempirical model," J. Appl. Meteorol. 30, 447-462 (1991).

30. See Ref. 15, pp. 306-310.

31. See Ref. 15 , pp. $224-225$.

32. See Ref. 15, pp. 256-259.

33. R. Perez, K. Webster, R. Seals, R. Stewart, and J. Barron, "Variations of the luminous efficacy of global and diffuse radiation and zenith luminance with weather conditionsdescription of a potential method to generate key daylight availability data from existing solar radiation data bases," Sol. Energy 38, 33-44 (1987).
34. J. A. Olseth and A. Skartveit, "Observed and modelled hourly luminous efficacies under arbitrary cloudiness," Sol. Energy 42, 221-233 (1989).

35. R. Perez, P. Ineichen, R. Seals, J. Michalsky, and R. Stewart, "Modeling daylight availability and irradiance components from direct and global irradiance," Sol. Energy 44 271-289 (1990).

36. B. Molineaux, P. Ineichen, and J. J. Delaunay, "Direct luminous efficacy and atmospheric turbidity-improving model performance," Sol. Energy 55, 125-137 (1995).

37. S. Pohlen, B. Ruck, and A. Bittar, "Evaluation of the Perez luminous efficacy models for a southern hemisphere site (New Zealand $\left.-41^{\circ} \mathrm{S}, 175^{\circ} \mathrm{E}\right)$," Sol. Energy 57, 307-315 (1996).

38. J. P. S. Parkinnen, J. Hallikainen, and T. Jaaskelainen, "Characteristic spectra of Munsell colors," J. Opt. Soc. Am. A 6, 318-322 (1989).

39. ASTM Committee E-12, "Standard practice for computing the colors of objects by using the CIE system (E 308-95), in ASTM standards on color and appearance measurements," (American Standards for Testing and Materials, Philadelphia, Pa., 1996), pp. 262-263.

40. The white point for the transformation to $L^{*} u^{*} v^{*}$ space was the standard illuminant $\mathrm{D}_{65}$. 